\title{
Corralito un Humedal en Vía de Extinción en el Corregimiento de Severá, Municipio de Cereté, Colombia
}

\section{Corralito: A Wetland in Danger of Extinction in the Village of Severá, Municipality of Cereté, Colombia}

\author{
Jorge Villadiego-Lorduy ${ }^{1}$ \\ Universidad Pontificia Bolivariana, Colombia \\ Lilliana Piedra-Castro ${ }^{2}$ \\ Universidad Nacional, Costa Rica \\ Hernán Tapia-Contreras ${ }^{3}$ \\ Yhonattan Méndez-Nobles ${ }^{4}$ \\ Omar Carmelo Díaz-Hernández ${ }^{5}$ \\ Universidad Pontificia Bolivariana, Colombia
}

http://dx.doi.org/10.15359/rgac.61-2.10

1 Doctor en Ciencias Naturales para el Desarrollo. Docente investigador del programa de ingeniería sanitaria y ambiental. Universidad Pontificia Bolivariana. Sede Montería, Colombia. Correo electrónico: Jorge.villadiegol@upb.edu.co

2 Doctora en Ciencias Naturales para el Desarrollo. Docente investigadora de la Escuela de Ciencias Biológicas de la Universidad Nacional, Costa Rica, sede Heredia. Correo electrónico: lilliana.piedra.castro@una.cr

3 Docente investigador del programa de ingeniería civil. Universidad Pontificia Bolivariana. Sede Montería, Colombia. Correo electrónico: Hernan.tapia@upb.edu.co

4 Docente investigador del programa de Ingeniería Sanitaria y Ambiental. Universidad Pontificia Bolivariana. Sede Montería, Colombia. Correo electrónico: Yhonattan.mendez@upb.edu.co

5 Docente investigador del programa de Ingeniería Sanitaria y Ambiental. Universidad Pontificia Bolivariana. Sede Montería, Colombia. Correo electrónico: omar.diazh@upb.edu.co

Fecha de recepción: 24 marzo de 2017

Fecha de aceptación: 31 de enero de 2018 
Jorge Villadiego-Lorduy, Lilliana Piedra-Castro, Hernán Tapia-Contreras, Yhonattan Méndez-Nobles, Omar Carmelo Díaz-Hernández. Corralito: A Wetland in Danger of Extinction in the Village of Severá, Municipality of Cereté, Colombia

\title{
RESUMEN
}

El propósito de este artículo es analizar las condiciones ambientales presentes en el humedal de Corralito, el cual se soportó en el análisis y características de las esferas social, económica y natural, a fin de entender las causas de los eventos físicos, económicos, sociales que expliquen los daños ambientales que ha sufrido en los últimos años. La problemática ambiental existente es el resultado de las presiones de tipo antrópico a la que ha sido sometido, la ausencia de una cultural ambiental y los intereses de los dueños de predios colindantes de apropiarse del espejo de agua lo han llevado a su desequilibrio y degradación. Como resultado del diagnóstico territorial, se identificaron dos problemas ambientales fundamentales: La desecación del humedal y la pérdida de la biodiversidad. Factores como el bajo nivel educativo, las escasas oportunidades de empleo, los pocos ingresos por núcleo familiar y las deficientes condiciones sanitarias no contribuyen al desarrollo sostenible local. Se resalta que un $80 \%$ del total de los núcleos familiares entrevistados tienen como actividad económica los oficios varios, y que la construcción de terraplenes y el proceso de parcelación contribuyó desde el año 2000 a una reducción drástica de su área.

Palabras clave: Problemas ambientales, fragmentación del ecosistema, dinámicas territoriales, presiones antrópicas, precarias condiciones socioeconómicas

\begin{abstract}
This article aims to analyze the environmental conditions present in the Corralito wetland, whose social, economic, and natural characteristics were considered to understand the causes of the physical, economic, and social events that explain the environmental damage it has suffered from in recent years. The existing environmental problem is the result of anthropogenic pressures to which it has been subjected, the absence of an environmental culture, and the interests of the owners of adjacent properties to appropriate the water mirror that have led to its imbalance and degradation. As a result of the territorial diagnosis, two fundamental environmental problems were identified: the drying of the wetland and the loss of biodiversity. Factors such as low educational level, scarce employment opportunities, low family income and poor sanitary conditions do not contribute to local sustainable development. It is highlighted that $80 \%$ of the families interviewed have diverse economic activities and that the construction of embankments and the parceling process contributed, since 2000 , to a drastic reduction of the wetland area.
\end{abstract}

Keywords: environmental problems, ecosystem fragmentation, territorial dynamics, anthropic pressures, precarious socio-economic conditions.

\section{Introducción}

Los humedales son ecosistemas que surgen cuando la saturación del terreno por agua produce sustratos hídricos (dominados por procesos anaeróbicos) y permite el establecimiento de biota, principalmente, plantas enraizadas, con adaptaciones para tolerar la anegación (Keddy, 2004); no solo son estaciones vitales en el itinerario del agua: son fuentes de biodiversidad (Instituto Alexander Von Humboldt, 2015), según Castellanos (2001), las ciénagas son los ecosistemas más productivos del mundo. Su característica determinante es la disposición constante o 
Jorge Villadiego-Lorduy, Lilliana Piedra-Castro, Hernán Tapia-Contreras, Yhonattan Méndez-Nobles, Omar Carmelo Díaz-Hernández. Corralito un Humedal en Vía de Extinción en el Corregimiento de Severá, Municipio de Cereté, Colombia

temporal de agua a lo largo de todo el año, esta situación favorece el desarrollo exitoso de una amplia diversidad de flora, fauna y microorganismos que interactúan en complejas relaciones para mantener un equilibrio ecológico de alta fragilidad.

Por otro lado, la problemática ambiental se ha acelerado y agudizado en las últimas décadas, hoy día la globalización económica impone nuevas pautas para la producción y consumo de recursos. Colombia no es ajena a tal situación, sobre todo para los ecosistemas tipo humedal, donde cada vez se van desecando y deteriorando sus condiciones ambientales. La región Caribe presenta diversos ambientes de aguas saladas y salobres que permiten la presencia, en esta zona, de la mayor variación (variedad) en cuanto a cuerpos de agua costeros del país se refiere, ellos son: ribereños, lacustres, palustres, marinos y estuarinos; de amplias playas arenosas y fondos lodosos. Forman parte de estos ecosistemas la ciénaga Grande del Magdalena, la desembocadura de los ríos Sinú y Atrato y la bahía de Cartagena. La extensión territorial va desde los límites con Panamá hasta los límites con Venezuela (aproximadamente $142.000 \mathrm{~km} 2$ ). Comprende localidades de los departamentos de la Guajira, Magdalena, Atlántico, Bolívar, Sucre, Córdoba y Antioquía, entre $7^{\circ} 56^{\prime}$ y $12^{\circ} 25^{\prime}$ latitud Norte y $77^{\circ} 20^{\prime}$ y $71^{\circ} 08^{\prime}$ longitud Oeste (Rangel et al., 1997).

La ciénaga de Corralito (aproximadamente a $10 \mathrm{msnm}$ ) está ubicada sobre la margen izquierda del río Sinú en su cuenca media, $54 \mathrm{~km}$ aguas arriba de su desembocadura, en las coordenadas N8 $52^{\prime} 39$ W75 $51^{\prime} 04$. Política y administrativamente hace parte del municipio de Cereté, corregimiento de Severá (Colombia). (Figura 1). 
Jorge Villadiego-Lorduy, Lilliana Piedra-Castro, Hernán Tapia-Contreras, Yhonattan Méndez-Nobles, Omar Carmelo Díaz-Hernández. Corralito: A Wetland in Danger of Extinction in the Village of Severá, Municipality of Cereté, Colombia

Figura 1. Localización de la ciénaga de Corralito

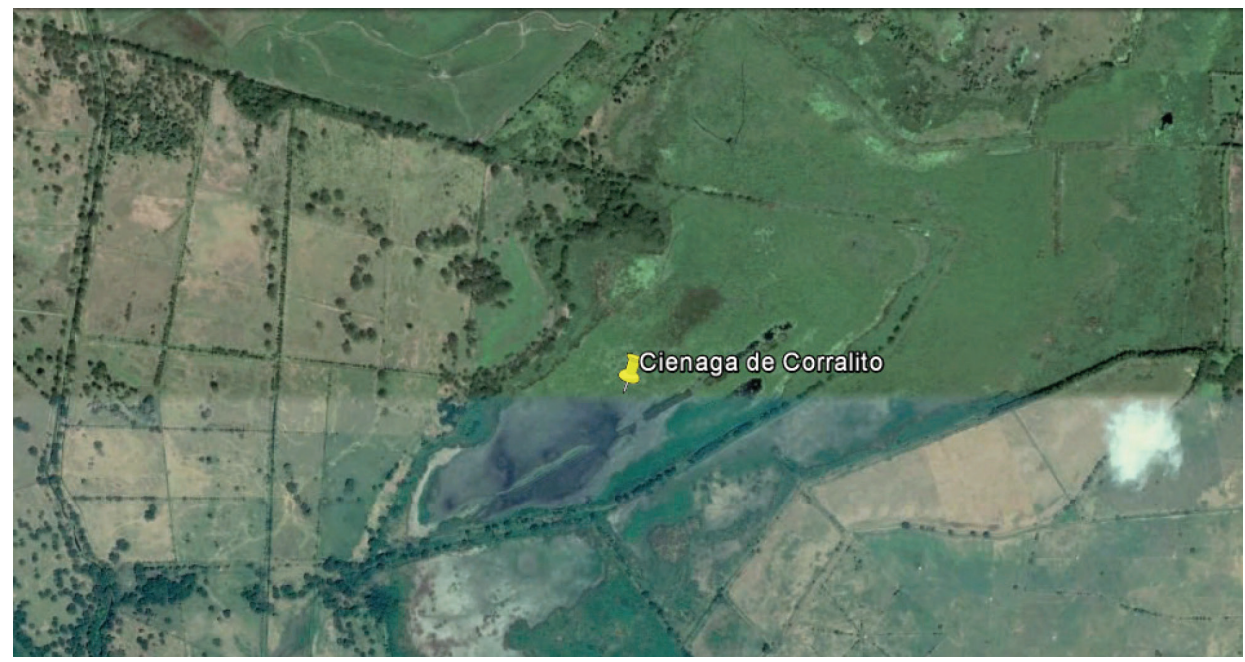

Fuente: Google Earth, 2016.

En el caso del humedal de Corralito, debido a la presión que ejercen sus habitantes, lo están llevando a sus límites ecológicos, lo anterior, originado por la demanda de recursos naturales. Por ello, con el objetivo de estudiar las condiciones ambientales de este ecosistema se desarrolló una valoración ambiental del espejo de agua y su población vecina, a fin de conocer el estado real en que se encuentran. La ausencia de una cultura ambiental que generen acciones positivas por parte de los habitantes hacia este, la construcción de terraplenes, procesos de parcelación, bajos ingresos económicos y nivel de educación son factores que están influyendo en su degradación. Además, se señala que a partir del análisis espacio - temporal para el espejo de agua, se determinó el punto exacto en el tiempo donde se aceleró su deterioro.

\section{Materiales y métodos}

El método que se utilizó en el estudio de las condiciones ambientales del humedal de Corralito se soportó en el análisis de información primaria y secundaria. En términos de información secundaria se recopiló reportes de proyectos ambientales desarrollados en el área de estudio (Diagnósticos y planes de manejo ambiental elaborados por la Corporación ambiental de los Valles del Sinú y San Jorge, 2016 y 2018; análisis socioeconómico 
Jorge Villadiego-Lorduy, Lilliana Piedra-Castro, Hernán Tapia-Contreras, Yhonattan Méndez-Nobles, Omar Carmelo Díaz-Hernández. Corralito un Humedal en Vía de Extinción en el Corregimiento de Severá, Municipio de Cereté, Colombia

ejecutado por Tapia y Pérez, 2016 entre otras investigaciones) y se desarrolló el respectivo análisis de imágenes de satélites tipo Landsat para los años de Enero de 1990, 1996, 2000 y 2015, mediante el software Arcgis 10.3, con el fin de conocer la dinámica espacio temporal que ha sufrido la ciénaga. Para el caso del estudio de la información primaria, se diseñó un instrumento de investigación tipo cuestionario que contenía 14 ítems, se aplicó a una muestra de 44 predios de un total de 70 predios del centro poblado de Severá (Sector Los Chorrillos). Se entrevistó a un representante de cada núcleo familiar existente en la muestra, cuando había más de un núcleo familiar por predio se seleccionaba al azar al individuo a entrevistar del núcleo familiar escogido; con la información primaria se buscó recopilar información de variables socioeconómicas, que permitieran contrastar con la información secundaria analizada, y con las visitas a campo realizadas al ecosistema en mención. Igualmente, para el procesamiento y análisis de la información recolectada de la aplicación del cuestionario se emplearon métodos de estadística descriptiva basada en el cálculo de estimaciones porcentuales. La información del total de predios existentes a la fecha y sobre la cual se trabajó el diseño muestral (Muestreo aleatorio simple) se obtuvo del plano predial del centro poblado del corregimiento (descargado desde el sitio web del Instituto Geográfico Agustín Codazzi en noviembre de 2016). El software con el que se elaboró el análisis estadístico fue $\mathrm{R}$ versión 3.0.2. Se asoció como técnica de investigación el trabajo de campo para la verificación de la problemática ambiental existente en el área de estudio, desarrollando las respectivas visitas a la zona en el mes de febrero de 2017.

\section{RESULTADOS Y DISCUSIÓN}

\section{Corralito y sus características físico-bióticas}

A nivel general, los suelos de la unidad donde se ubica la Ciénaga de Corralito constituyen un relieve plano y micro relieve ondulado con pendientes usualmente menores a 1 (0-3\%). Geomorfológicamente, el humedal se puede caracterizar como una ciénaga dulce, que hace parte de un complejo de zonas inundables y zonas encharcables de carácter permanente y de régimen natural, que están delimitadas al Oeste por los palocauces del valle del Sinú, como son Caño Viejo, Caño Vidrial o Caño Caimanera, 
las principales geoformas presentes son basin alto, bajo, delta de explayamiento y manto de desborde. El espejo de agua y su área de influencia se ubican sobre una litología conformada por capas de grava, arena, limo y arcilla depositadas en un ambiente fluvio-lacustre que definen un acuífero discontinuo de extensión local libre a semiconfinado de baja productividad (Corporación de los Valles del Sinú y San Jorge, 2008).

La Corporación de los Valles del Sinú y San Jorge en el Plan De Manejo y Gestión Ambiental de los Humedales Corralito, Martinica, Pantano Largo y Pantano Grande, desarrollado en el 2008, indica que las precipitaciones tienen valores totales anuales que oscilan entre $826 \mathrm{y}$ $1278 \mathrm{~mm}$. Los valores medios mensuales varían entre $11 \mathrm{~mm}$, (Estación Galán), y 197 mm (Estación Cereté), ocurridos durante el mes de agosto. La temperatura media anual varía entre 25.7 y $29.7^{\circ} \mathrm{C}$. Los valores más altos de temperatura media mensual se presentan durante los meses de marzo y abril y las más bajas en los meses de octubre a diciembre; sin embargo, la variación de temperatura mensual no supera los $5^{\circ} \mathrm{C}$. Para la ciénaga se estima la humedad relativa media multianual en $80.8 \%$, una velocidad de viento media multianual de $1.15 \mathrm{~m} / \mathrm{s}$ y la evaporación media multianual de $2200 \mathrm{~mm} / \mathrm{año}$. Los valores totales anuales de brillo solar son en promedio de 2.710 horas. El mes de mayor brillo solar es enero con 261.1 horas/mes y el de menor es septiembre con 211.3 horas/mes. Según el plan de manejo, la zona del humedal y alrededores se puede clasificar (clasificación de Holdridge) en un clima cálido a semicálido y desde el punto de vista de flora y fauna la cobertura vegetal presenta 6 categorías florísticas enmarcadas en: 1) Agua y vegetación flotante, 2) Zona de transición 3) Matorrales y bosque redictual, 4) Bosque ripario, 5) Pastizal y 6) Agroecosistemas (Gráfico 1). 
Jorge Villadiego-Lorduy, Lilliana Piedra-Castro, Hernán Tapia-Contreras, Yhonattan Méndez-Nobles, Omar Carmelo Díaz-Hernández. Corralito un Humedal en Vía de Extinción en el Corregimiento de Severá, Municipio de Cereté, Colombia

Gráfico1. Distribución de la diversidad de especies y géneros de vegetación según hábitat

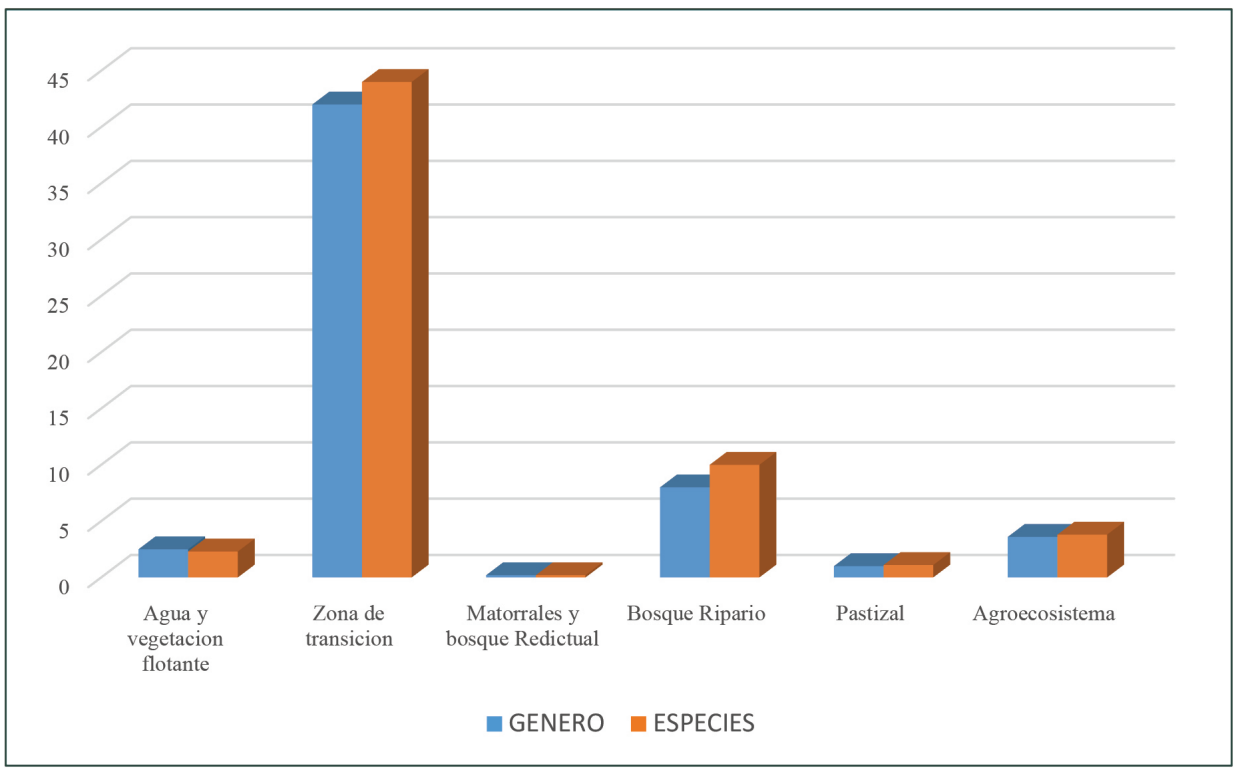

Fuente: Corporación Ambiental de los Valles del Sinú y San Jorge (CVS, 2008).

Para el ambiente acuático se cuenta con especies vegetales como lechuga de agua (Pistia stratiotes) y buchón de agua (Eichhornia azurea), en lo referente a la zona transicional, se caracteriza por presentar vegetación capaz de resistir inundaciones de más de un metro de profundidad se cuenta con zarza o dormidera" (Mimosa pigra) y "bocachica" (Thalia geniculata), Altamisa" (Ambrosia peruviana), "Enea o Nea" "Junco" (Typha angustifolia), y Pimiento" (Phyllanthus cf. acuminatus). En el ambiente terrestre coexisten especies como algarrobillo" o "campano" (Samanea saman); le sigue en importancia el "ñipi-ñipi" (Sapium aucuparium), el muñeco (Cordia sp., cf. panamensis) el "roble rosado" (Tabebuia rosea) y el "chengue" (Erythrina glauca). Dentro de los árboles subordinados se encuentran el "naranjuelo" (Crataeva tapia), el "guácimo" (Guazuma ulmifolia) y en menor proporción, el "espino" (Pithecellobium lanceolatum) con Andira inermis. Dentro de las especies de pastos la especie predominante es gramalote "Paspalum sp y Panicum maximum, por último, y no menos importante, están las coberturas conformadas por cultivos, ya sea de pancoger 
Jorge Villadiego-Lorduy, Lilliana Piedra-Castro, Hernán Tapia-Contreras, Yhonattan Méndez-Nobles, Omar Carmelo Díaz-Hernández. Corralito: A Wetland in Danger of Extinction in the Village of Severá, Municipality of Cereté, Colombia

o tecnificados, cuya diversidad está dada por el uso, es decir, coberturas vegetales derivadas de los agroecosistemas. Por otro lado, la fauna que es característica de este tipo de ecosistemas se centra en tres categorías:

1. Herpetofauna conformada por Anura (Ranas y Sapos) con 13 especies, Sauria (reptiles) con 16 especies, Serpentes (serpientes) con 17 especies, testudines (tortugas) con 3 especies y Crocodylia (cocodrilos) con 1 especie.

2. Aves, se encontró un total de 86 especies de Aves, repartidas en 36 familias, 82 géneros; La familia más representativa fue Tyranidae (pequeños pájaros) con la mayor cantidad de especies (10) seguida por la Familia Ardeidae (aves pelicaniformes, garzas) con 8 especies y las familias Accipitridae (aves rapaces, gavilanes), Anátidae (patos) e ictáridae (turpiales) con 5 especies cada una.

3. Mamíferos, la mayor riqueza de especies se concentra en los murciélagos de la familia Phylostomidae, el ponche o cacó (Hydrochaeris hydrochaeris). Los marsupiales didélfidos (Orden Didelphimorphia) representan el 7,6\% de las especies de mamíferos presentes en el área de estudio. La familia Didelphidae está representada por la chucha o fara (Didelphis marsupialis). De los mamíferos carnívoros restantes, continúan presentes el zorro perro (Cerdocyon thous) y zorra piemuchacho (Procyon cancrivorus), también se cuenta en menor proporción con la presencia monos aulladores (Alouatta seniculus).

Hay que aclarar que debido a acelerado proceso de degradación de la ciénaga muchas especies tanto de fauna como flora han desaparecido al no contar con las condiciones mínimas para su subsistencia (Tapia y Pérez, 2016), para la fauna; especies como el venado (Mazama americana y Odocoileus virginianus) y el zaíno (Pecari tajacu) ya han desaparecido, en el caso del armadillo (Dasypus novemcinctus) este es muy escaso. Igualmente, ocurrió con grandes gatos (jaguar, panthera onca, y el puma, puma concolor) que fueron parte de la fauna de la región (Corporación de los Valles del Sinú y San Jorge, 2008). Por lo tanto, Corralito ha enfrentado, debido a las presiones de orden antrópico y tal como lo señala Badii et al (2015) un proceso de extinción de especies a nivel local, en la que la 
Jorge Villadiego-Lorduy, Lilliana Piedra-Castro, Hernán Tapia-Contreras, Yhonattan Méndez-Nobles, Omar Carmelo Díaz-Hernández. Corralito un Humedal en Vía de Extinción en el Corregimiento de Severá, Municipio de Cereté, Colombia

especie deja de existir en un área determinada, aunque sigue viviendo en otro lugar. Este fenómeno también es conocido como extirpación.

\section{Importancia ecológica de Corralito}

La ciénaga de Corralito, en primera medida es uno de los humedales más representativos del municipio de Cereté, el hecho que este ecosistema de vital importancia para el municipio se encuentre en sus límites de subsistencia por la ignorancia de vecinos, terratenientes de la región y por la falta de autoridad ambiental para el cumplimiento de la normatividad (El Meridiano de Córdoba, 2016), ha generado una serie de implicaciones ambientales dentro de las que se señalan:

- Al no existir el humedal, se acaba con el hábitat de muchas especies de animales y plantas que depende de este tipo de ecosistemas. Al alterar su tamaño, hidrología, patrones de vegetación afecta el stock de alimento disponible para comunidades de aves, las cuales tienen como hábitat las ciénagas (Blanco, 1999). Hay que recordar que el régimen hidrológico es reconocido como el principal factor condicionante de las propiedades estructurales y funcionales de los ecosistemas de humedal (Mitch y Gosselink 2000).

- $\quad$ Se prescinde de los servicios ecológicos que presta el cuerpo de agua en sus diferentes escalas territoriales: Local, regional, nacional y global. Los humedales contribuyen a la regulación de los microclimas, sin omitir que son patrimonio de la humanidad, en tal caso no se pueden vender o negociar ni mucho menos apropiarse de los mismos. Igualmente, los humedales aportan a la manutención de la calidad del agua, reteniendo sedimentos y nutrientes, y la remoción de tóxicos, además de brindar una serie de recursos naturales útiles al hombre para desarrollar actividades productivas.

- $\quad \mathrm{Al}$ desaparecer Corralito se perdería una importante reserva de agua para la región, comprometiendo las condiciones de vida de las comunidades que se benefician de este espejo de agua. Así mismo, los humedales son el sostén de la cultura de muchos pueblos, en especial de aquellos que tienen arraigada la cultura del agua y lo referente a su gobernanza. 
Jorge Villadiego-Lorduy, Lilliana Piedra-Castro, Hernán Tapia-Contreras, Yhonattan Méndez-Nobles, Omar Carmelo Díaz-Hernández. Corralito: A Wetland in Danger of Extinction in the Village of Severá, Municipality of Cereté, Colombia

\section{Características socioeconómicas y percepciones de la población vecina del humedal}

A partir del análisis de los resultados obtenidos con la aplicación del cuestionario de caracterización socioeconómica de los núcleos familiares entrevistados en el centro poblado del corregimiento de Severá (Sector los chorrillos), se estima que un $80 \%$ de las familias viven del rebusque diario, es decir, actividades como el mototaxismo, "un día de jornal", albañilería, reparaciones, entre otras, son las que le brindan un ingreso económico a las familias, no siendo dichas labores bien remuneradas ni estables. El restante $20 \%$ se divide en un $2 \%$ dedicado a la pesca y el otro $18 \%$ se dedican a actividades como la agricultura (Gráfico 2). Para Boyce (citado por Correa, 2007) las desigualdades sociales y económicas son factores determinantes en la explicación de la calidad ambiental.

Gráfico 2. Actividad económica del área de estudio.

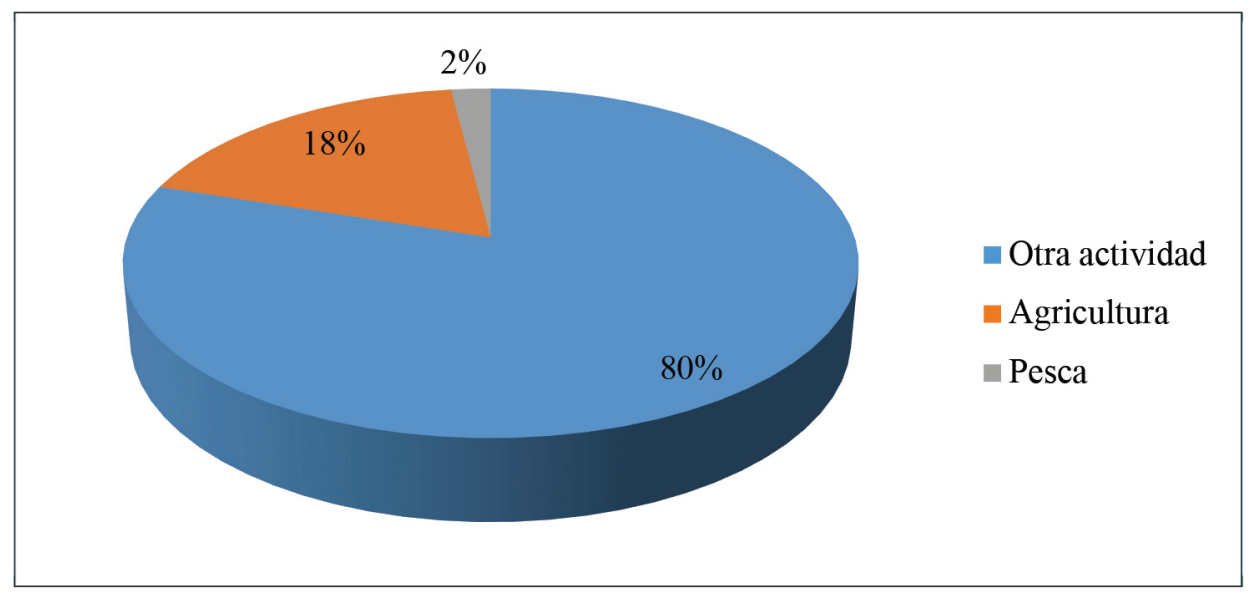

Fuente: Elaboración propia producto de análisis estadístico

En términos de educación, es importante destacar que a pesar de la iniciativa del gobierno de aumentar el nivel educativo en Colombia, en muchas zonas del país se presentan altos índices de analfabetismo y la zona de estudio no es la excepción, $62 \%$ de los hogares encuestados cuentan con al menos un individuo con estudios secundarios incompletos, un $2 \%$ cuentan por lo menos con una persona que no sabe leer ni escribir, y solo un 36\% de los entrevistados cursaron estudios de primaria completos (Gráfico 3). 
Jorge Villadiego-Lorduy, Lilliana Piedra-Castro, Hernán Tapia-Contreras, Yhonattan Méndez-Nobles, Omar Carmelo Díaz-Hernández. Corralito un Humedal en Vía de Extinción en el Corregimiento de Severá, Municipio de Cereté, Colombia

Gráfico 3. Nivel de educación en el área de estudio

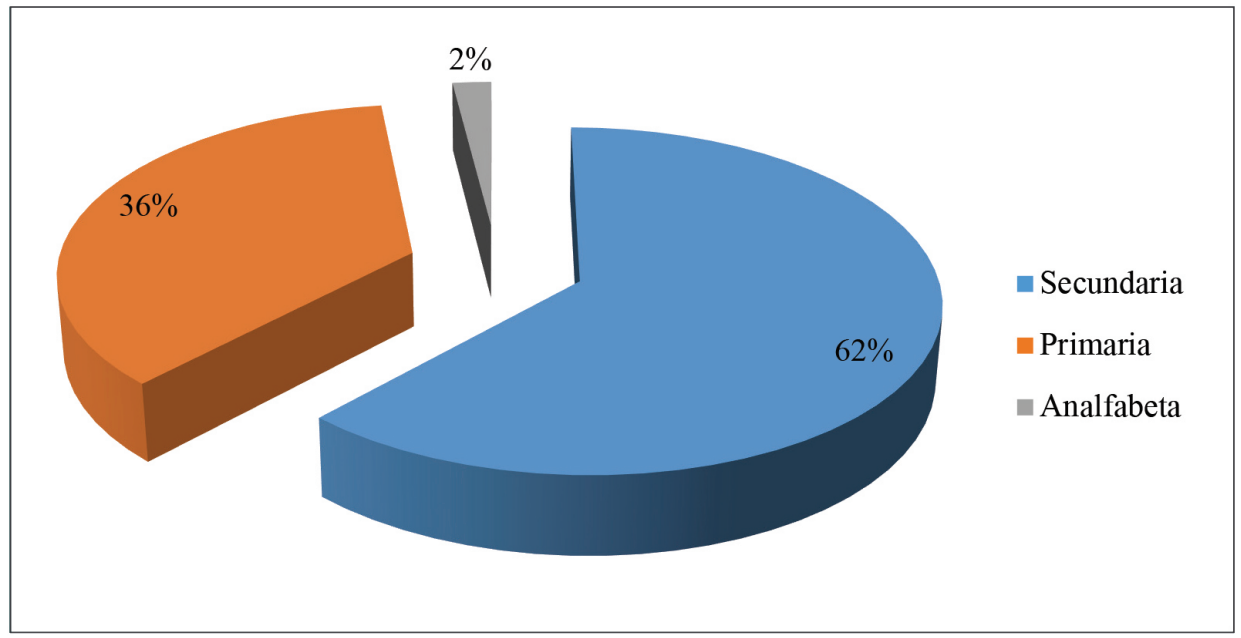

Fuente: Elaboración propia producto de análisis estadístico

En cuanto a la infraestructura se refiere, el $67.9 \%$ de la población rural del municipio de Cereté, incluyendo Severá, no ha logrado satisfacer sus necesidades básicas, el corregimiento no cuenta con servicios de alcantarillado ni con el de recolección de basuras, éstas son quemadas o enterradas. El servicio de energía eléctrica tiene una cobertura del 95\%. El corregimiento carece del servicio de gas natural, siendo el fogón de leña y la pimpina de gas propano las opciones más viables para la cocción de los alimentos. La cobertura del acueducto es del $60 \%$, su administración está a cargo de una junta comunitaria y el agua es bombeada directamente del rio sin ningún tratamiento previo (Corporación Ambiental de los Valles del Sinú y San Jorge, 2008). Referente a las viviendas y los materiales que las conforman, en el área rural las paredes pueden ser de bareque, guadua, madera o zinc y piso de tierra, en el casco urbano las paredes son de bloques con su respectivo repello, y pisos de cemento o baldosa.

$\mathrm{Al}$ respecto de la organización comunitaria en el corregimiento de Severá, al preguntarle a los entrevistados si conocían de alguna organización al interior de la comunidad de carácter no gubernamental que trabajaran de forma articulada por el bienestar local, señalaron las siguientes: Juntas de Acción Comunal, Madres Comunitarias, ASOPAS, AMIPROS, Grupo Deportivo el Chorrillo, Asociación de Aguas de Severá, APROPESCA y ASPROACER. 
Por otra parte, en cuanto a las percepciones que tienen los pobladores del humedal, el $100 \%$ de los entrevistados para época lluviosa, lo consideran un proveedor de alimento, es decir, suministra frutas y la producción de peces se reanuda por las lluvias y el flujo de agua proveniente como proceso natural por parte del río Sinú. El mismo porcentaje de encuestados en verano, solo lo percibe como un territorio de tránsito para llegar a diferentes lugares. Un 98\% de los entrevistados le confieren importancia al humedal como ecosistema vital para la zona, pero la pregunta que surge es si este ecosistema es considerado relevante para las poblaciones vecinas ¿Por qué han permitido su degradación? Prácticamente es muy poco lo que queda de este cuerpo de agua, entonces no existe coherencia entre las respuestas dadas y las acciones de las personas hacia la ciénaga.

\section{Dinámica espacial y problemática ambiental de la ciénaga de Corralito}

Para el análisis de los cambios espacio-temporales ocurridos sobre el espejo de agua, se trabajó con una imagen Lansat TM5 y tres imágenes Lansat ETM7, tomadas en el mismo mes de cada año, enero de 1990 (Lansat 5), 1996, 2000 y 2015 con las que se desarrolló mediante el software ARCGIS versión 10.3 el respectivo análisis espacial para cada periodo mencionado. Hacia 1990, el ecosistema presentaba una planicie aluvial donde se daba un proceso de intercambio de aguas entre la ciénaga, los Caños Viejo y la Caimanera (drenaje cercano al espejo de agua) y el río Sinú, es decir, el humedal se encontraba hidráulicamente activo, alrededor del espejo de agua central se contaba con pequeñas ciénagas que servían de área de amortiguamiento, no se apreciaba ningún tipo de fragmentación ecosistémica en la zona producto de actividad antrópica, y el área total del espejo de agua era en ese entonces de 3720 ha. La circulación y energía de la ciénaga era notable con los cuerpos de aguas aledaños, lo cual indicaba la conectividad hídrica y la abundancia de coberturas de agua para las poblaciones aledañas al humedal (figura 2). 
Jorge Villadiego-Lorduy, Lilliana Piedra-Castro, Hernán Tapia-Contreras, Yhonattan Méndez-Nobles, Omar Carmelo Díaz-Hernández. Corralito un Humedal en Vía de Extinción en el Corregimiento de Severá, Municipio de Cereté, Colombia

Figura 2. Perfil del Valle aluvial que muestra los diferentes reservorios de aguas superficiales.

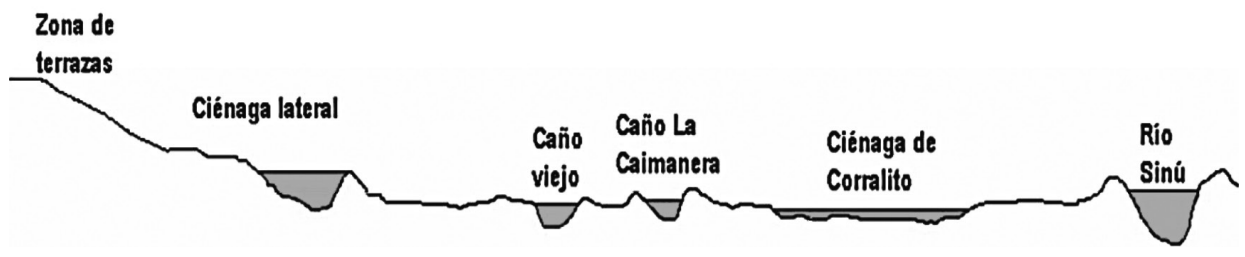

Fuente: Conservación Internacional, 2008.

Hacia 1996, el área del cuerpo de agua se redujo a 193,7 ha, alterándose su hidrología en términos de taponamiento de caños e interconectividad con el río y la desecación de algunas zonas de amortiguamiento, se perciben algunos procesos de deterioro de la ciénaga asociado a la desecación de las zonas antes mencionadas. En el año 2000 ocurre un cambio drástico en la estructura ecológica del ecosistema, dándose tres fenómenos que alteran el territorio, el primero asociado con la construcción de terraplenes, con un total para ese año de 56 estructuras de este tipo, correspondiente a un total longitudinal de $58,8 \mathrm{Km}$. El segundo fenómeno, igualmente importante, fue la parcelación de zonas perimetrales de Corralito, aproximadamente se parcelaron 1200 ha. Lo anterior originó un proceso de fragmentación del ecosistema dividiendo los terrenos y el espejo de agua en diversos sectores, perdiendo así comunicación interna y externa el ecosistema en su conjunto. Según García (2011), la fragmentación de ecosistemas está asociado a tres procesos de alteración paisajística: 1) la pérdida progresiva de superficie de hábitat original; 2) la subdivisión creciente del hábitat remanente; y 3) el incremento de la relación perímetro/ superficie en dicho hábitat.

Por último, no se puede omitir el cambio de uso del suelo como consecuencia de la parcelación y construcción de terraplenes, se pasó teniendo en cuenta el decreto 3600 de 2007 artículo 1, acerca de la reglamentación de los usos del suelo rural, de un uso de estructura ecológica principal a un uso prohibido, es decir, un uso incompatible con el uso principal de una zona, con los objetivos de conservación ambiental y de planificación ambiental y territorial, y por consiguiente implicación de graves riesgos de tipo ecológico y/o social (Corporación de los Valles del Sinú y San Jorge, 2016). Para el año 2000 el área del espejo de agua era de 98,8 ha, 
Jorge Villadiego-Lorduy, Lilliana Piedra-Castro, Hernán Tapia-Contreras, Yhonattan Méndez-Nobles, Omar Carmelo Díaz-Hernández. Corralito: A Wetland in Danger of Extinction in the Village of Severá,

Municipality of Cereté, Colombia

reduciéndose en un $51 \%$ en comparación con el año 1996 y en un $97,5 \%$ referente a 1990. En el 2015 se da la desaparición total del espejo de agua (Únicamente se puede apreciar en imágenes una huella hídrica de 9 ha), apareciendo solo en periodos de lluvia, se conserva a lado y lado de los terrenos de la ciénaga cultivos transitorios y vegetación típica del humedal: Enea (Typha angustifolia), se da un cambio total del uso del suelo a agropecuario y se han legalizado con escritura pública y matricula inmobiliaria mucho de los terrenos pertenecientes al humedal (figuras 3 y 4 ). 
Jorge Villadiego-Lorduy, Lilliana Piedra-Castro, Hernán Tapia-Contreras, Yhonattan Méndez-Nobles, Omar Carmelo Díaz-Hernández. Corralito un Humedal en Vía de Extinción en el Corregimiento de Severá, Municipio de Cereté, Colombia

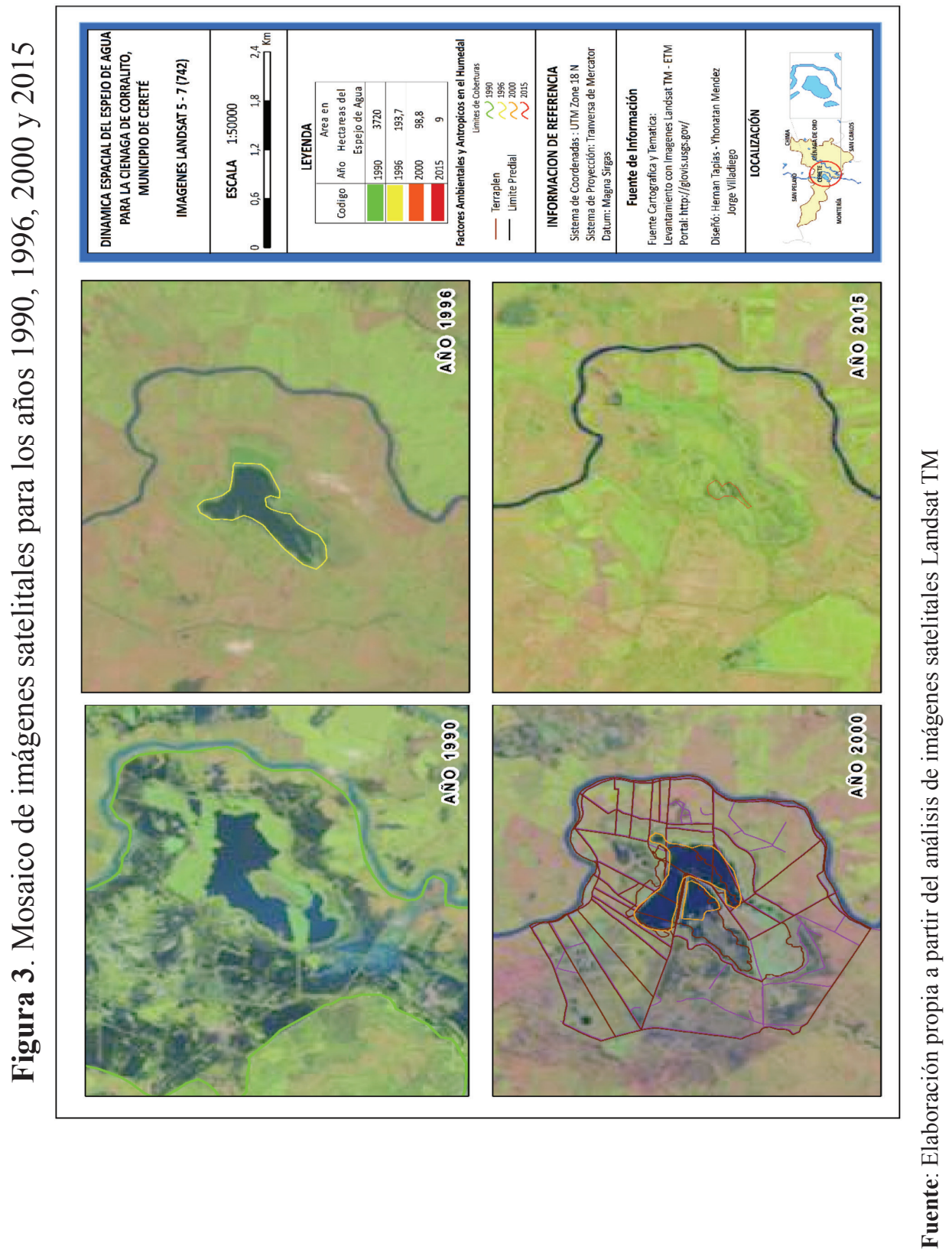


Jorge Villadiego-Lorduy, Lilliana Piedra-Castro, Hernán Tapia-Contreras, Yhonattan Méndez-Nobles, Omar Carmelo Díaz-Hernández. Corralito: A Wetland in Danger of Extinction in the Village of Severá, Municipality of Cereté, Colombia

Figura 4. Mapa de dinámica del espejo de agua para los años 1990, 1996,2000 y 2015

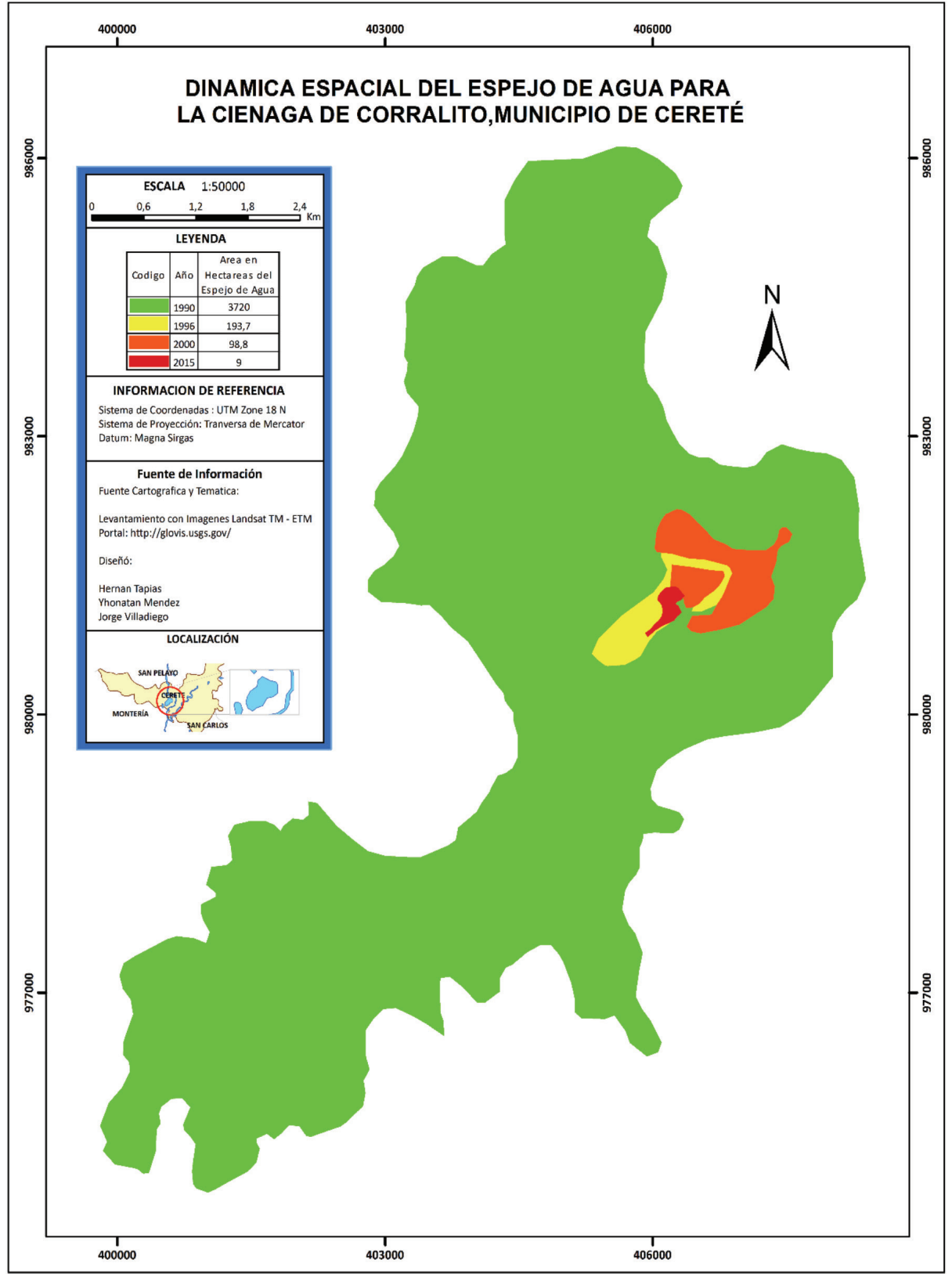

Fuente: Elaboración propia a partir del análisis de imágenes satelitales Landsat TM 
Jorge Villadiego-Lorduy, Lilliana Piedra-Castro, Hernán Tapia-Contreras, Yhonattan Méndez-Nobles, Omar Carmelo Díaz-Hernández. Corralito un Humedal en Vía de Extinción en el Corregimiento de Severá, Municipio de Cereté, Colombia

Así mismo, la desecación del humedal se convierte en una de las principales problemáticas ambientales de Corralito, derivada de la construcción de terraplenes y la parcelación de los terrenos (fotos 1 y 2). Seguidamente, la pérdida de la biodiversidad producto de la fragmentación del ecosistema y la caza de especies que todavía se encuentran en la zona, de igual forma, la falta de una cultura ambiental de los vecinos más próximos a esta dificulta su conservación, sin omitir las actividades agrícolas y pecuarias que desarrollan los dueños de las fincas aledañas al humedal (Cuadro 1). Desafortunadamente, para la ciénaga los bosques han sido destruidos, principalmente, para dar lugar a zonas de actividades agropecuarias y para la explotación selectiva de maderables de gran valor comercial o para el consumo de leña, sin que existan áreas de uso combinado productivo-forestal. Como consecuencia de lo anterior, las poblaciones de especies de flora y fauna han disminuido drásticamente mientras que otras ya han desaparecido, de allí que el estado ambiental de la ciénaga sea muy crítico (Corporación Ambiental de los Valles del Sinú y San Jorge, 2008). Hay que recordar que los humedales son extremadamente sensibles a los cambios del medio ambiente terrestre y de su cuenca, y que esos cambios en algunos casos son imposibles de revertir (Convención relativa a los Humedales de Importancia Internacional, 2007).

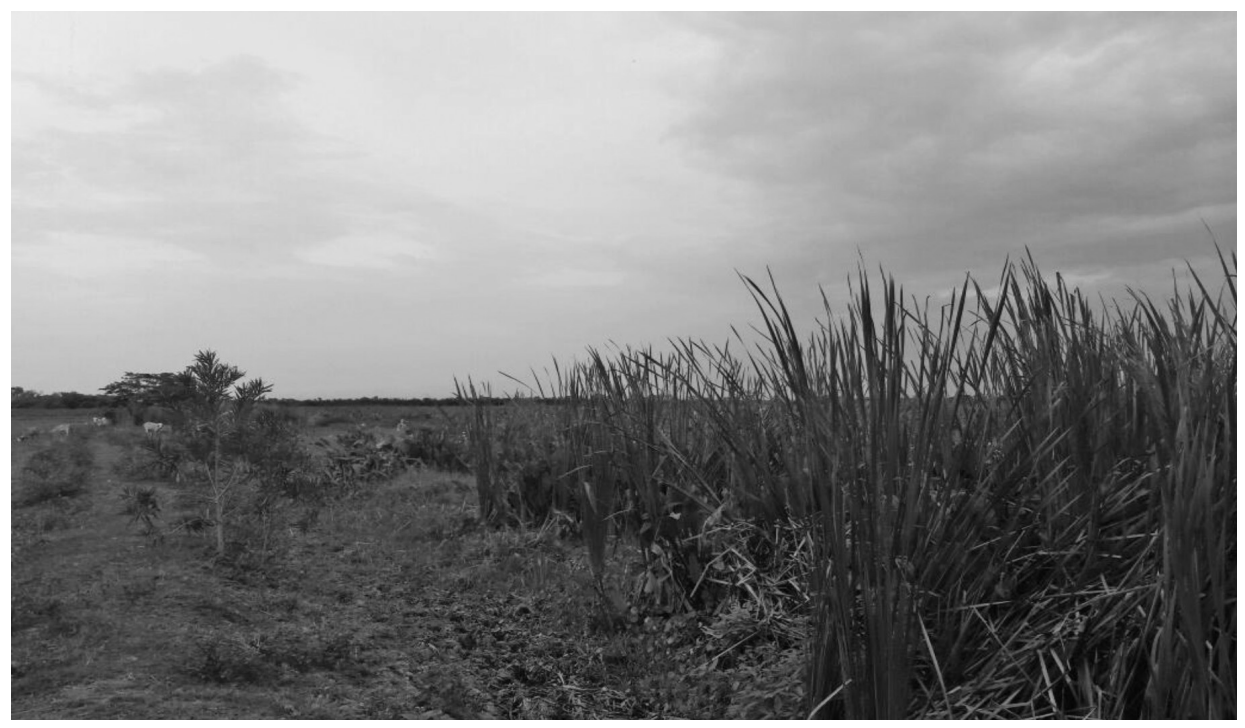

Foto 1. Terraplén y vegetación de Enea (Typha angustifolia) 
Jorge Villadiego-Lorduy, Lilliana Piedra-Castro, Hernán Tapia-Contreras, Yhonattan Méndez-Nobles, Omar Carmelo Díaz-Hernández. Corralito: A Wetland in Danger of Extinction in the Village of Severá, Municipality of Cereté, Colombia

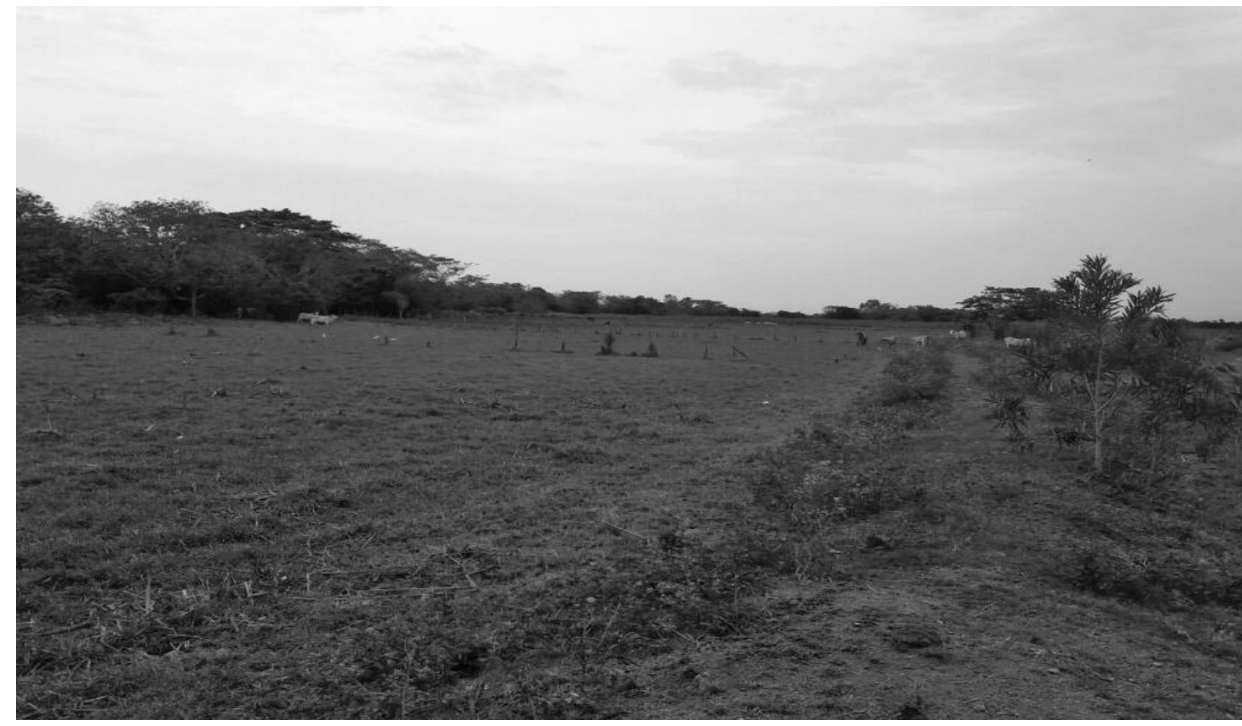

Foto 2. Terraplén como elemento de fragmentación del humedal

Dentro de los actores involucrados en la problemática ambiental descrita, se tienen actores institucionales como la Corporación ambiental de los Valles del Sinú y San Jorge(CVS), la Policía Ambiental y la Alcadia de Cereté, como principales actores sociales se encuentran las comunidades vecinas de los humedales, y como actores económicos y de gran relevancia en el deterioro de los humedales se tiene a los agricultores y ganaderos que han ayudado a la extinción del humedal con la construcción de terraplenes para expandir sus predios. Se necesitan actores en el área de estudio, que desde la gestión ambiental y tal como lo propone Rodríguez y Espinosa (2003, p.10) identifiquen la problemática del ambiente, y formulen e implementen políticas dirigidas a su protección. 
Jorge Villadiego-Lorduy, Lilliana Piedra-Castro, Hernán Tapia-Contreras, Yhonattan Méndez-Nobles, Omar Carmelo Díaz-Hernández. Corralito un Humedal en Vía de Extinción en el Corregimiento de Severá, Municipio de Cereté, Colombia

Cuadro 1. Matriz de problemas ambientales ciénaga de Corralito

\begin{tabular}{|c|c|c|c|c|}
\hline \multicolumn{5}{|c|}{ MATRIZ DE PROBLEMAS AMBIENTALES } \\
\hline $\begin{array}{l}\text { PROBLEMAS } \\
\text { AMBIENTALES }\end{array}$ & CAUSA & ЕFECTO & $\begin{array}{c}\text { NIVEL DE } \\
\text { IMPORTANCIA }\end{array}$ & ACTORES \\
\hline \multirow{4}{*}{$\begin{array}{l}\text { Desecación del } \\
\text { humedal }\end{array}$} & Eutrofización & $\begin{array}{l}\text { Sedimentación del } \\
\text { fondo del humedal }\end{array}$ & \multirow{4}{*}{ Alto } & \multirow{4}{*}{$\begin{array}{l}\text { Comunidad, } \\
\text { Autoridad } \\
\text { ambiental (CVS), } \\
\text { Policía ambiental, } \\
\text { Alcaldía, } \\
\text { Ganderos y } \\
\text { agricultores }\end{array}$} \\
\hline & \multirow{3}{*}{$\begin{array}{l}\text { Construcción } \\
\text { de terraplenes y } \\
\text { parcelación }\end{array}$} & $\begin{array}{l}\text { Carencia de } \\
\text { interconexión hídrica } \\
\text { entre los cuerpos de } \\
\text { agua }\end{array}$ & & \\
\hline & & $\begin{array}{l}\text { Pérdida de las áreas del } \\
\text { humedal }\end{array}$ & & \\
\hline & & $\begin{array}{l}\text { Cambio de usos del } \\
\text { suelo y transformación } \\
\text { del paisaje }\end{array}$ & & \\
\hline \multirow{5}{*}{$\begin{array}{l}\text { Pérdida de la } \\
\text { biodiversidad }\end{array}$} & $\begin{array}{l}\text { Caza indiscriminada } \\
\text { y pesca con artes no } \\
\text { adecuadas }\end{array}$ & \multirow{5}{*}{ Destrucción de habitats } & \multirow{5}{*}{ Alto } & \multirow{5}{*}{$\begin{array}{l}\text { Comunidad, } \\
\text { Autoridad } \\
\text { ambiental (CVS), } \\
\text { Policía ambiental, } \\
\text { Alcaldía, } \\
\text { Ganderos y } \\
\text { agricultores }\end{array}$} \\
\hline & $\begin{array}{l}\text { Fragmentación del } \\
\text { ecosistema }\end{array}$ & & & \\
\hline & $\begin{array}{l}\text { Explotación } \\
\text { agropecuaria }\end{array}$ & & & \\
\hline & $\begin{array}{l}\text { Ausencia de una } \\
\text { cultura ambiental }\end{array}$ & & & \\
\hline & Tala de árboles & & & \\
\hline
\end{tabular}

Fuente: Elaboración propia derivado de trabajo de campo.

\section{Conclusiones}

Con el desarrollo del estudio de las condiciones ambientales del humedal de Corralito se concluye lo siguiente:

- Las características socioeconómicas de la principal población vecina del humedal son críticas, bajos niveles de educación y actividades económicas relacionadas con la informalidad y los bajos ingresos económicos son foco del poco desarrollo local que presenta la zona.

- La infraestructura del corregimiento de Severá en término de cobertura de servicios públicos, excepto el de energía, es mínimo, ausencia del servicio de recolección de basuras, gas y alcantarillado debido a la poca gestión comunitaria y de la administración de turno.

- Desde 1996 se empieza a alterar la hidrología de la ciénaga de Corralito con el taponamiento de caños y desecación de pequeños humedales aledaños al espejo de agua estudiado, pero es en el año 2000 
Jorge Villadiego-Lorduy, Lilliana Piedra-Castro, Hernán Tapia-Contreras, Yhonattan Méndez-Nobles, Omar Carmelo Díaz-Hernández. Corralito: A Wetland in Danger of Extinction in the Village of Severá, Municipality of Cereté, Colombia

donde se intensifica la construcción de terraplenes, seguida de la parcelación de los terrenos pertenecientes al humedal y se desata la crisis ambiental del humedal.

- Los principales problemas ambientales del humedal se centran, principalmente, en la desecación del humedal por la construcción de terraplenes y procesos de parcelación por parte de los dueños de fincas, y en la pérdida de la biodiversidad y extinción local de algunas especies como efecto del mismo proceso de fragmentación del ecosistema por los factores antes mencionados.

- La poca articulación entre la autoridad ambiental, policía ambiental, alcaldía y comunidad ha brindado los espacios para que terceros (terratenientes) se lucren con el humedal apropiándose y legalizando los terrenos robados al ecosistema.

\section{Recomendaciones}

A partir del análisis de las condiciones socioambientales y del proceso de deterioro del humedal de Corralito se realizan las siguientes recomendaciones:

- Generar un modelo de desarrollo sostenible local que responda a las particularidades del territorio y del cual se logren empoderar las comunidades residentes aledañas al humedal convirtiéndose dicho modelo en un pilar para el desarrollo de las mismas.

- Desarrollar procesos de educación ambiental con las comunidades vecinas del humedal y asociar dicho proceso a proyectos económicos y de autogestión de organizaciones comunitarias.

- Comprometer a las autoridades locales, regionales y nacionales con la recuperación del humedal y la declaratoria de área protegida para la conservación de especies y hábitats, además de empezar el proceso de deslinde de los terrenos pertenecientes al humedal.

- Implementar mecanismos y/o estrategias de seguimiento y evaluación para el modelo de desarrollo sostenible local y los procesos de educación ambiental que se adelanten en los territorios de Corralito. 
Jorge Villadiego-Lorduy, Lilliana Piedra-Castro, Hernán Tapia-Contreras, Yhonattan Méndez-Nobles, Omar Carmelo Díaz-Hernández. Corralito un Humedal en Vía de Extinción en el Corregimiento de Severá, Municipio de Cereté, Colombia

\section{Referencias}

Badii, M. H., Guillen, A., Pérez, G., Aguilar, J., UANL, S. N. D. L. G., \& México, N. L. (2015). Extinción de Especies y su Implicación Species Extinction and its Implication. Daena: International Journal of Good Conscience, 10(1), 157-171. Consultado en Mar 10 de 2017. Recuperado de http://www.spentamexico.org/v10-n1/A11.10(1)157-171.pdf

Blanco, D. E. (1999). Los humedales como hábitat de aves acuáticas. Tópicos sobre humedales subtropicales y templados de Sudamérica. Oficina Regional de Ciencia y Tecnología de la UNESCO para América Latina y el Caribe-ORCYT-Montevideo-Uruguay, 219-228.

Castellanos, C. (2001). Los ecosistemas de humedales en Colombia. Revista Luna Azul. 13(7):45-56. . Consultado en Mar 20 de 2017. Recuperado de http://lunazul.ucaldas.edu.co/downloads/Lunazul13_4.pdf

Convención relativa a los Humedales de Importancia Internacional especialmente como Hábitat de Aves Acuáticas (Ramsar). (2006). Manual de la convención de Ramsar: Guía a la convención sobre los humedales. Consultado en Mar 15 de 2017.

Corporación Ambiental de los Valles del Sinú y San Jorge. (2008). Plan de manejo y gestión ambiental de los humedales Corralito, Martinica, Pantano largo y Pantano Grande.

Corporación Ambiental de los Valles del Sinú y San Jorge. (2016). CVS Inició Recuperación de Distrito de Conservación de Suelo Ciénaga de Corralito Cereté. Consultado en enero 23 de 2018. Recuperado de http://cvs.gov.co/web/cvs-inicio-recuperacion-de-distrito-de-conservacion-de-suelo-cienaga-de-corralito-cerete/

Correa, F. (2007). Crecimiento económico, desigualdad social y medio ambiente: evidencia empírica para América Latina. Ingenierías, 6 (1). Consultado en mar 23 de 2017. Recuperado de http://www.scielo.org. $\mathrm{co} /$ scielo.php?script=sci_arttext\&pid=S1692-33242007000100002

El Meridiano de Córdoba. (2016). Ciénaga de corralito, un "falso positivo". Consultado en enero 23 de 2018. Recuperado de http://www. elmeridiano.co/cienaga-de-corralito--un-falso-positivo/35997

García, D. (2011). Efectos biológicos de la fragmentación de hábitats: nuevas aproximaciones para resolver un viejo problema. Revista Ecosistemas, 20(2-3). Consultado en marzo 5 de 2017. Recuperado de http://revistaecosistemas.net/index.php/ecosistemas/article/view/18 
Jorge Villadiego-Lorduy, Lilliana Piedra-Castro, Hernán Tapia-Contreras, Yhonattan Méndez-Nobles, Omar Carmelo Díaz-Hernández. Corralito: A Wetland in Danger of Extinction in the Village of Severá, Municipality of Cereté, Colombia

Instituto Alexander Von Humboltdt. (2015). Colombia anfibia: Un país de humedales. Instituto Humboldt, Editores. ISBN obra impresa: 978958-8889-48-1 ISBN obra digital: 978-958-8889-47-4

Keddy, P. A. (2004). Wetland Ecology. Cambridge University Press, Cambridge

Mitch, W. J. y Gosselink, J. (2000). Wetlands. Third Edition. John Wiley and Sons. New York 920pp.

Rangel, J., Lowy, D. y Aguilar, M. (1997). Distribución de los tipos de vegetación en las regiones naturales de Colombia. En Colombia Diversidad Biótica II 383-402. Bogotá: Universidad Nacional de Colombia.

Rodríguez, B. y Espinosa, G. (2003). Gestión Ambiental en América Latina y el Caribe: Evolución, tendencias y principales prácticas. Consultado en Mar 25 de 2017. Recuperado de http://www.iadb.org/sds/ publication/publication_3351_s.htm

Secretaría de la Convención de Ramsar (2013). Manual de la Convención de Ramsar: Guía a la Convención sobre los Humedales (Ramsar, Irán, 1971) (6a. ed.). Secretaría de la Convención de Ramsar, Gland (Suiza). Recuperado de www.ramsar.org/sites/default/files/documents/library/manual6-2013-sp.pdf

Tapia, H. y Pérez, N. (2016). Diseño de estrategias de educación ambiental para el manejo sostenible de la Ciénaga de Corralito, municipio de Cereté-Córdoba. Revista Escenarios 19:42-49. Consultado en enero 23 de 2018. Recuperado de http://revistas.cecar.edu.co/escenarios/ article/view/297 